\title{
Receptivity for a Flat Plate with a Rounded Leading-Edge
}

\author{
D.E. Nichols and P.W. Hammerton \\ University of East Anglia, Norwich NR4 7TJ, UK
}

\begin{abstract}
The receptivity due to the interaction of an acoustic wave with a body which has a rounded leading edge and a region of low wall shear is considered. The body of interest is formed by a line source in a uniform stream. The nose radius, $r_{n}^{*}$, of this body is characterised in the theory through a parameter $A=$ $2 \omega r_{n}^{*} / 3 U_{\infty}$ where $\omega$ is the frequency of the acoustic wave and $U_{\infty}$ the mean flow speed. By comparing asymptotic and numerical results, the receptivity coefficient, $C_{1}$, is calculated. The receptivity coefficient is seen to decrease dramatically with a small increase in nose radius, a local rise occurs about $A=0.035$ and it then gradually declines to nearly zero when $A=0.1$.
\end{abstract}

\section{Introduction}

Receptivity is the process by which external disturbances generate instability waves in the boundary layer. Regions where receptivity occurs are those that exhibit rapid streamwise variations in the mean boundary layer flow or sudden changes in the surface boundary conditions. In this paper, leading-edge receptivity to acoustic waves is considered for a body which has a region of low wall shear close to the leading edge. The flow is assumed to be twodimensional, irrotational and with high Reynolds number. The body considered has a rounded leading edge with continuous surface curvature and tends to a flat plate and is chosen so that the inviscid slip velocity is known in analytic form. The acoustic wave is considered to have a single frequency and to be a small perturbation to the free-stream, giving the inviscid slip velocity as

$$
U_{s}^{*}(s, t)=U_{f s}{ }^{*}(s)+\varepsilon U_{d}^{*}(s) e^{-i \omega t^{*}},
$$

where $\varepsilon \ll 1$.

In the development of the unsteady boundary layer flow there exists two distinct streamwise regions whose solutions overlap $[1,2]$. In the first region the linearised unsteady boundary layer equation (LUBLE) is satisfied and in the second the motion is governed by the Orr-Sommerfeld equation. In the overlap region the asymptotic eigensolutions of the LUBLE match onto the Tollmien-Schlichting waves in the matched asymptotic sense. The lowest order of these eigensolutions matches onto the spatially growing TollmienSchlichting wave, and the receptivity coefficient, $C_{1}$, is considered to be this eigensolution's coefficient. 


\section{Formulation of Equations and Body Geometry}

The dimensional boundary layer equations in coordinates $(s, n)$, where $s$ is measured along the body, and $n$ normal to it, are non-dimensionalised by introducing the typical velocity, length and time scales which are $U_{\infty}, U_{\infty} / \omega$, $\omega^{-1}$ respectively. The stream function $\psi$ is then introduced and following the process used to determine self-similar equations, it is assumed that $\psi$ takes the form $\psi=h(s) \phi(s, N, t)$ where $N=g(s) n$. The dimensionless boundary layer equation then becomes

$$
\begin{aligned}
\phi_{N t}+\frac{d(g h)}{d s} \phi_{N}^{2} & +g h\left(\phi_{N} \phi_{N s}-\phi_{N N} \phi_{s}\right) \\
& =\frac{1}{g h}\left(\frac{\partial U_{s}}{\partial t}+U_{s} \frac{\partial U_{s}}{\partial s}\right)+g^{2} \phi_{N N N}+g \frac{d h}{d s} \phi \phi_{N N},
\end{aligned}
$$

with the usual boundary conditions. To retrieve terms similar to those appearing in the Blasius' equation requires setting $g=h^{\prime}$. The outer boundary condition implies $g h=U_{f s}$ and $\phi_{N} \rightarrow 1+\varepsilon\left(U_{d} / U_{f s}\right) e^{-i t}$ as $N \rightarrow \infty$. Solving for $g, h$ gives

$$
g(s)=U_{f s}\left\{2 \int U_{f s} d s\right\}^{-1 / 2} \quad h(s)=\left\{2 \int U_{f s} d s\right\}^{1 / 2} .
$$

Writing $\phi(s, N, t)=\phi_{1}(s, N)+\varepsilon \phi_{2}(s, N) e^{-i t}+O\left(\epsilon^{2}\right)$, substituting into (2) gives a nonlinear equation for the steady flow,

$$
\begin{aligned}
\phi_{1 N N N}+\phi_{1} \phi_{1 N N} & =f 1(s)\left(\phi_{1 N}^{2}-1\right)+f 2(s)\left(\phi_{1 N} \phi_{1 N s}-\phi_{1 N N} \phi_{1 s}\right) \\
\text { where } \quad f 1(s) & =\frac{2 \int U_{f s} d s}{U_{f s}{ }^{2}} \frac{d U_{f s}}{d s}, \quad f 2(s)=\frac{2 \int U_{f s} d s}{U_{f s}},
\end{aligned}
$$

and a linear equation for the perturbation flow. Comparing the equation for $\phi_{1}$ with the standard equation governing the evolution of a steady boundary layer,

$$
f_{N N N}+f f_{N N}=\beta(\xi)\left(f_{N}^{2}-1\right)+2 \xi\left(f_{N} f_{N \xi}-f_{N N} f_{\xi}\right)
$$

a change of variables needs to occur in (4) such that

$$
f 2(s) \frac{\partial}{\partial s}=2 \xi \frac{\partial}{\partial \xi} \quad \Rightarrow \quad \xi=k \int_{0}^{s} U_{f s}\left(s^{\prime}\right) d s^{\prime},
$$

where $k$ is an arbitrary constant and is chosen to be unity. The function $f 1(s)$ takes on the role of the mean pressure gradient, $\beta(\xi)$. This change of variables in the equation for $\phi_{2}$ gives the LUBLE,

$$
\begin{aligned}
\phi_{2 N N N} & +\phi_{2 N N}\left\{\phi_{1}+2 \xi \phi_{1 \xi}\right\}+\phi_{2 N}\left\{i f 3(\xi)-2 \beta(\xi) \phi_{1 N}-2 \xi \phi_{1 N \xi}\right\} \\
& +\phi_{2} \phi_{1 N N}+2 \xi\left\{\phi_{1 N N} \phi_{2 \xi}-\phi_{1 N} \phi_{2 N \xi}\right\} \\
= & \frac{U_{d}}{U_{f s}}\{i f 3(\xi)-\beta(\xi)\}-\frac{2 \xi}{U_{f s}} \frac{d U_{d}}{d \xi}, \quad f 3(\xi)=\frac{2 \xi}{U_{f s}{ }^{2}} .
\end{aligned}
$$


To construct a body with the properties described in $\S 1$, we consider the flow due to a line source with strength $\kappa$ within a uniform stream. The non-dimensional complex velocity potential is

$$
\Phi=z+A \log \left(\frac{U_{\infty}}{\omega}\right)+A \log (z), \quad \text { where } \quad A=\frac{\kappa \omega}{2 \pi U_{\infty}^{2}} .
$$

A stagnation point occurs at $z=-A$ and the streamlines are given by $\psi=y+A \arg (z)$. Replacing the stagnation point streamline with a rigid body gives a body described by $x=-y \cot (y / A)$ where $-A \pi<y<A \pi$. Figure 1 compares this body of interest (abbreviated to USLS) with a parabola and a modified super ellipse (MSE), bodies which have been studied experimentally and numerically $[2,3]$. The non-dimensional nose radius of the body is $r_{n}=3 A / 2$ with thickness tending to $2 A \pi$. Thus, the parameter $A$ can be thought of either as an indicator of nose radius or the frequency of the disturbance. The inviscid slip velocity $U_{f s}(s)$ is known implicitly through $y$ :

$$
\begin{aligned}
U_{f s}(y) & =\left\{1+\frac{A^{2} \sin ^{2}(y / A)}{y^{2}}-\frac{A \sin (2 y / A)}{y}\right\}^{1 / 2}, \\
\frac{d s}{d y} & =\left\{1+\left(-\cot (y / A)+\frac{y}{A} \operatorname{cosec}^{2}(y / A)\right)^{2}\right\}^{1 / 2} .
\end{aligned}
$$

\section{Asymptotic Solutions $(\xi \rightarrow \infty)$}

Letting $\bar{\xi}=\xi / A$, the steady equation to be solve can be seen to be independent of $A$ and thus independent of the free stream disturbance, as required. The mean pressure gradient $\beta(\bar{\xi})$, starts favourable, becomes adverse then tends to zero far downstream. The effect of this adverse pressure gradient is to reduce the steady wall shear, however, as seen in figure 2, for this body it is insufficient to produce boundary layer separation. Far downstream the wall shear takes the form

$$
\phi_{1 N N}(\bar{\xi}, 0) \sim F^{\prime \prime}(0)\left\{1+\alpha \frac{\ln (\bar{\xi})}{\bar{\xi}}+\frac{\gamma}{\bar{\xi}}\right\}+O\left(\bar{\xi}^{-1.887}\right),
$$

where $\mathrm{F}$ is the Blasius function, $\alpha=-1.2023$ is found by a solvability condition through adjoint operators, and the $\gamma=4.711$ is found numerically. The next order term is due to eigensolutions found by Libby \& Fox [5].

The asymptotic solution of the LUBLE takes the form

$$
\phi_{2} \sim \phi_{2 S t}(\xi, N ; A)+\sum_{i} C_{i}(A) \phi_{2 i}(\xi, N ; A),
$$

where $\phi_{2 S t}$ is a generalisation of the Stokes shear wave, determined locally, and $\phi_{2 i}$ are eigensolutions, analogous to those obtained by Lam \& Rott [4] for the flat plate. These eigensolutions are solutions of (7) with the forcing set 
to zero. To find the eigensolutions' asymptotic form requires the asymptotic form of $f 3$ and $\beta$, which is why the slip velocity is required in functional form.

As $\xi \rightarrow \infty$ the eigensolutions develop a two-layer behaviour, the inner layer of width $O\left(\xi^{-1 / 2}\right)$ and the main layer of $O(1)$. In the inner layer the variables $G=(2 \xi)^{1 / 2} \phi_{2}$ and $m=(2 \xi)^{1 / 2} N$ are introduced, and the $i t h$ eigensolution takes the form,

$$
\begin{aligned}
\phi_{2 i} & =(2 \xi)^{\left(\tau_{i}-1 / 2\right)} e^{T_{i}(\xi)}\left\{p_{i}(M)+(2 \xi)^{-3 / 2} q_{i}(M)+\ldots\right\}, \\
T_{i}(\xi) & =-\frac{\lambda_{i}(2 \xi)^{3 / 2}}{F^{\prime \prime}(0)}\left\{\frac{1}{3}-A \alpha \frac{\ln (\xi / A)}{\xi}+\frac{A}{\xi} \bar{\gamma}\right\}+O\left(A^{1.887} \xi^{-0.387}\right), \\
\tau_{i} & =-\left(\frac{889-16 \rho_{i}^{3}}{1260}\right)-i \frac{A}{\left[F^{\prime \prime}(0)\right]^{2}}\left(\frac{8 \rho_{i}^{3}-27}{10 \rho_{i}^{3}}\right),
\end{aligned}
$$

where $M=(1-A / \xi) m, \bar{\gamma}=(2 \alpha-\gamma-3), p_{i}(M)$ is a known function, $\rho_{i}$ satisfies $\operatorname{Ai}^{\prime}\left(-\rho_{i}\right)=0$, and the eigenvalues $\lambda_{i}$ are found analogous to the flat plate $([1])$ and given by $\lambda_{i}=\rho_{i}^{-3 / 2} e^{-\pi i / 4}$. The constants $\alpha, \gamma$ are those that appear in the asymptotic solution of $\phi_{1},(11)$. The coefficients, $C_{i}$ of the eigensolutions have to be determined numerically.

\section{Numerical Solution}

The receptivity coefficient $C_{1}$ is found by comparing the numerical solution of the LUBLE (7) integrated from $\xi=0$, with the asymptotic form of the first eigensolution. In this current work, a uniform pulsating stream parallel to the body is considered thus $U_{d}$ is set equal to $U_{f s}$.

The eigensolutions decay exponentially with distance downstream with the first eigensolution decaying faster than those of higher order. This situation can be reversed by moving the calculations into a segment of the complex plane, such that $\Re(T)>0$. When taking the solution into the complex plane, a contour must first be chosen in terms of $y$ due to the implicit expression for the slip velocity. Problems arise in the numerical solution when the steady wall shear approaches zero. To avoid this difficulty, the contour is taken along the real axis past the point of minimum wall shear, and then moved into the complex plane. It is then extended in such a way that the $\xi$-contour progresses into the required segment for exponential growth. In addition, the complex $y$-contour must terminate at $(A \pi, 0)$ to ensure that $|\xi| \rightarrow \infty$.

Figure 3 shows the absolute value of the receptivity coefficient, $C_{1}$, with change in $A$. It can be seen that the maximum value of $C_{1}$ for this body occurs at $A=0$ and corresponds to that found for the flat plate. As $A$ increases the receptivity initially decreases rapidly, with a local rise about $A=0.035$, and then falls off to nearly zero by $A=0.1$. As $A$ increases, the accuracy of the solution gradually reduces.

It was found that a fine spatial resolution of the numerical grid was required along the real axis (so that information was not lost on the eigensolutions, which decay exponentially for real $\xi$ ), and in particular near the steady 
wall shear minimum. The fine resolution was also necessary when $y \rightarrow A \pi$, as a small change in $y$ in this limit would correspond to a large change in $\xi$.

\section{Conclusions}

The receptivity of a stream-lined body to freestream acoustic disturbances has been addressed. The body geometry was chosen to be similar to that used in experimental and numerical studies, but such that the slip velocity could be determined analytically. The receptivity process involves unsteady forcing of the boundary layer in the vicinity of the leading edge, and gives rise to boundary layer disturbances whose wavelengths shorten as they propagate downstream. It is the eigensolutions of the unsteady solution that provides this shortening length scale, with one eigensolution matching onto the spatially growing Tollmien-Schlichting disturbance. Here, we choose the coefficient of that particular eigensolution, $C_{1}$, as our measure of receptivity.

For the body of interest, a symmetric mean flow with an acoustic wave propagating parallel to it has been considered. Figure 3 shows the receptivity coefficient as a function of the parameter $A=2 r_{n}^{*} \omega / 3 U_{\infty}$ where $r_{n}^{*}$ is dimensional nose radius. It is seen that an increase in nose radius decreases the receptivity coefficient. Work on the parabola ([2]) also demonstrated this, but with a small rise for very small nose radii which is not seen here. To compare the results, if $A=2 S / 3$ where $S$ is the Strouhal number used in [2], then $r_{n}^{*}$ is the same for both bodies. For the body considered here, the receptivity coefficient is almost zero at $A=0.1$, which corresponds to $S=0.15$ where $C_{1} \approx 0.57$ for the parabola. Thus, this body appears to reduce the receptivity much faster with increase in nose radius than the parabola.

A larger receptivity coefficient means that transition may be expected earlier. However, the transition position also depends on the stability characteristics of the steady flow. For a parabolic body the mean pressure gradient is always favourable, so the boundary layer is stabilised as the nose radius is increased. For the body of this study (as well as the MSE), the pressure gradient is favourable close to the nose, but adverse further downstream. Thus the drop in receptivity coefficient as nose radius increases is counteracted by a decrease in the stability of the boundary layer further downstream.

The experimental results of Saric et al [3] for modified super-ellipses, with aspect ratios $1: 20$ and $1: 40$ correspond to $A=0.006$ and $A=0.002$. These values of $A$ are marked on figure 3 , however direct comparison of asymptotic results with experimental results is difficult at the present time due to the use of different definitions of receptivity.

\section{References}

1. Goldstein, M.E. (1983) The evolution of Tollmien - Schlichting waves near a leading edge. J. Fluid Mech. 127, 59-81 
2. Hammerton, P.W. \& Kerschen, E.J. (1995) Boundary-layer receptivity for a parabolic leading edge. J. Fluid Mech. 310, 243-267

3. Saric, W.S., Wei, W., Rasmussen, B.K. \& Krutckhoff, T.K (1995) Experiments on leading edge receptivity to sound. AIAA 95-2253

4. Lam, S.H. \& Rott, N. (1960) Theory of linearized time dependant boundary layer equations. Cornell Univ. Grad. School of Aero. Engng Rep. AFORSR TN60-1100

5. Libby, P.A. \& Fox, H. (1963) Some perturbation solutions in laminar boundary layer theory. Part 1. The momentum equation. J. Fluid Mech. 17, 433-449

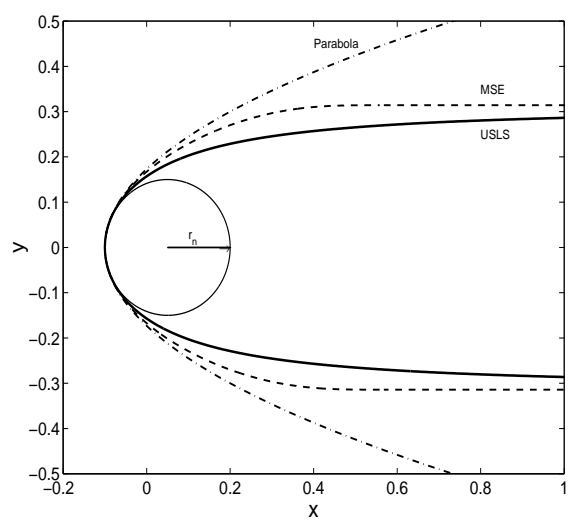

Figure 1: A comparison of the USLS (solid line), parabola (dashed line) and MSE (dash-dot line)

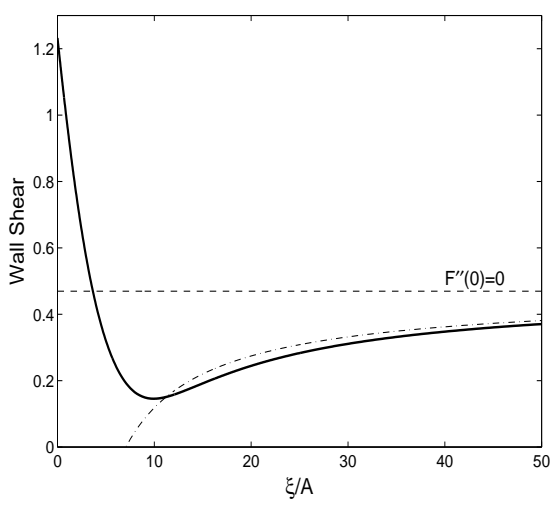

Figure 2: Numerical solution of steady wall shear (solid line) and the asymptotic solution (dashed line)

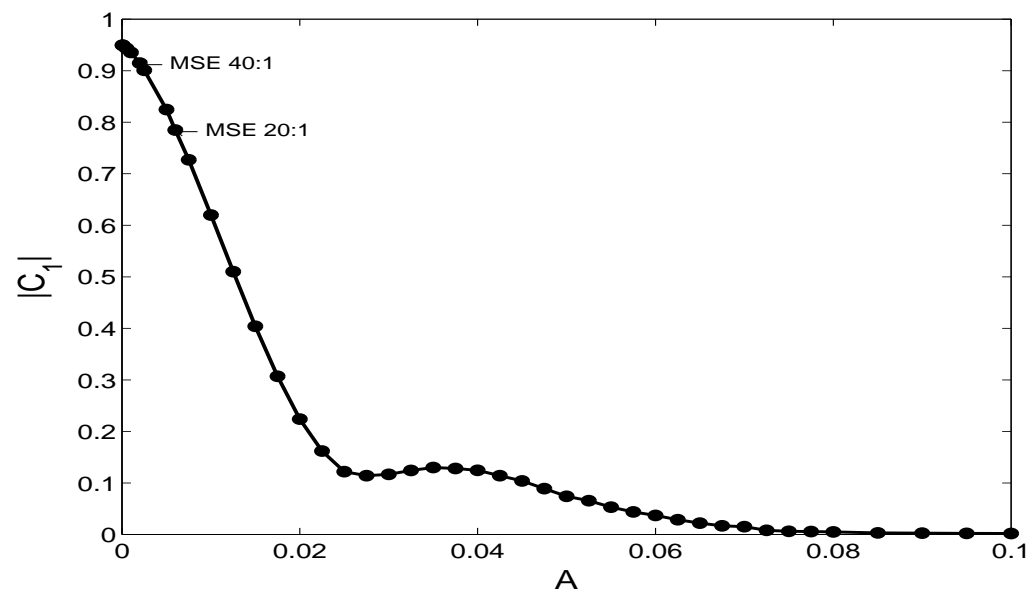

Figure 3: Change in receptivity coefficient, $C_{1}$, with $\mathrm{A}$ 